\title{
Steroid-induced ptosis: case studies and histopathologic analysis
}

A Song, KD Carter, JA Nerad, C Boldt and

J Folk

\begin{abstract}
Purpose The purpose of this study was to review patients who developed ptosis after subtenon's steroid injection and to study the mechanism of steroid-induced ptosis in an animal model.
\end{abstract}

Methods Part 1. Twenty-two patients with uveitis who had received posterior subtenon's triamcinolone acetonide injections were retrospectively reviewed. Demographics, type of uveitis, type and number of surgeries, pre and postoperative marginal reflex distance (MRD1), and clinical outcomes were evaluated. Part 2. Study of rabbit levator muscle and aponeurosis histopathology after subtenon's triamcinolone injection was performed.

Results Part 1. The average age was 44.6 years (range: $14-85$ years) with a mean followup of 14 months. The most common causes of uveitis included uveitis after cataract extraction (five), pars planitis (three), multifocal choroiditis (three), and juvenile rheumatoid arthritis (three). The average time to documented onset of ptosis was 13.9 months (range: 0-49 months). In patients who received only one steroid injection, the average time to ptosis recorded was 2.7 months (range: 0-6 months). Seventeen patients underwent ptosis repair. Part 2. No ptosis was noted in the experimental and control groups.

Histopathologic analysis of levator tissues revealed no significant difference in atrophy or degree of inflammation between experimental and control groups.

Conclusions Ptosis following subtenon's steroid injection ranged from mild to moderate and occurred a few months after steroid injection. Prior studies of muscles and periocular tissues exposed to corticosteroids demonstrated degenerative muscle changes; our studies revealed no histopathologic changes in the levator muscle or aponeurosis.
Eye (2008) 22, 491-495; doi:10.1038/sj.eye.6702667; published online 12 Janaury 2007

Keywords: ptasis; Subtenonis steroid injection; histopathology

Introduction

Corticosteroids are potent medications

commonly used to decrease ocular inflammation. However, a variety of adverse ocular and systemic side effects can occur with the use of periocular steroids, including decreased wound strength, orbital fat atrophy, decreased ocular motility. ${ }^{1-4}$

A common indication for periocular steroid injections is the treatment of uveitis. Posterior subtenon's injections of triamcinolone acetonide (TAA) allow a high concentration of long-acting steroids to be placed close to the posterior aspect of the globe; this is useful in decreasing cystoid macular oedema in patients with posterior uveitis. Complications include transient elevation in intraocular pressure and mild persistent ptosis. ${ }^{5-9}$

It is well-known that steroids result in muscle atrophy. ${ }^{10}$ However, no histopathologic studies of levator muscle or of the levator aponeurosis in these cases have been reported. The aim of this study is to describe the clinical findings in a series of patients who developed ptosis following posterior subtenon's injections of TAA and to evaluate the histopathology of eyelid tissues following steroid injection in an animal model.

Materials and methods

Part 1

After approval of the study by the Institutional Review Board, a retrospective review of all patients at the University of Iowa Hospitals \& Clinics between January 1984 and December 2004 who developed ptosis following subtenon's steroid injection was performed. These were performed by five ophthalmologists
University of lowa Hospitals \& Clinics, Department of Ophthalmology, lowa City, IA, USA

Correspondence: JA Nerad, University of lowa Hospitals \& Clinics, 200 Hawkins Drive, PFP, lowa City, IA 52242, lowa, USA

Tel: + 1319356 2590;

Fax: + 13193560363

E-mail: Jeffrey-nerad@ uiowa.edu

Received: 27 February 2006 Accepted in revised form: 30 October 2006 Published online: 12 Janaury 2007 
in the department, although most of the injections were administered by two ophthalmologists (CF, JF). After a drop of proparacaine and application of $4 \%$ lidocaine to the superotemporal conjunctiva on a cotton-tipped pledget for a few minutes, an eyelid speculum was placed under the upper and lower eyelids. One cc of $40 \mathrm{mg} / \mathrm{cc}$ TAA (Kenalog-40, Bristol-Myers Squibb, Princeton, NJ, USA) was injected into the superotemporal quadrant of subtenon's space with a $11 / 2$ inch 25 -gauge needle. Patient demographics, type of uveitis, type and number of surgeries, pre- and postinjection marginal reflex distance (MRD1), time from steroid injection to the date ptosis was first noted, and outcomes were evaluated.

\section{Part 2}

After Institutional Animal Care and Use Committee review and approval, four New Zealand white male rabbits (weight range: 4-6 lbs) were purchased by Harlan Sprague (Indianapolis, IN, USA). Each of the rabbits was anaesthetized with ketamine $50 \mathrm{mg}$ and acepromazine $5 \mathrm{mg}$. One cc of kenalog $(40 \mathrm{mg} / \mathrm{cc})$ was injected in the superotemporal quadrant of subtenon's space of the left eye. The subtenon's space in the fellow eye (right eye) was injected with $1 \mathrm{cc}$ of normal saline as a control (Figure 1). After 18 days, the animals were euthanized with euthasol $1 \mathrm{cc}(390 \mathrm{mg}$ pentobarbital, $50 \mathrm{mg}$ phenytoin) by injection into the cardiac muscle. The levator muscle and aponeurosis of the upper eyelids were harvested (Figure 2) and placed in paraformaldehyde. Light microscopy was performed on tissue sections.

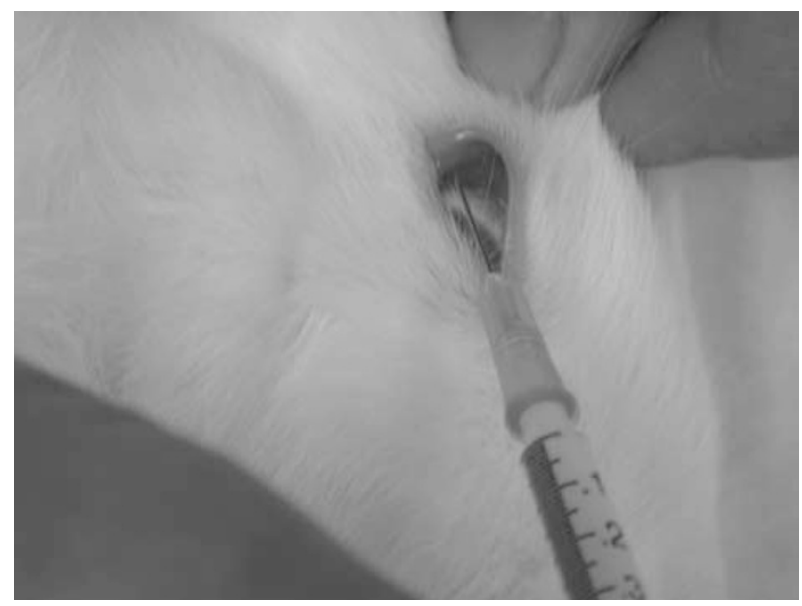

Figure 1 Triamcinolone acetonide injection. Injection of TAA was performed in the superotemporal quadrant in subtenon's space in the New Zealand rabbit.

\section{Results}

Part 1

Of over 900 subtenon's steroid injections, 22 patients had documented ptosis that prompted referral for evaluation. There were 15 females and seven males. The average age was 44.6 years (range: $14-85$ years, median 43 , mean 27 ). Thirteen right eyes and nine left eyes were involved.

Steroid injections were administered for cystoid macular oedema secondary to the following: uveitis after cataract extraction (five), pars planitis (three), multifocal choroiditis (three), juvenile rheumatoid arthritis (three), HLA-B27 uveitis (two), histoplasmosis (two), AZOOR (one), Birdshot uveitis (one), psoriatic uveitis (one), recurrent uveitis (one).

Twenty-one patients had received only sub-tenon's kenalog injection. One patient had received both subtenon's and subconjunctival steroid injections. Six (27\%) patients had received only one steroid injection treatment before developing ptosis. These procedures were performed with similar techniques by five vitreoretinal surgeons, with the majority of the injections by two of the vitreoretinal surgeons (CB, JF). Eight (36\%) patients had received two steroid treatments, and nine $(41 \%)$ had received more than two steroid treatments. On average, ptosis was noted 13.9 months (range: 0-49, median: 6 months) after the first steroid treatment. In those patients who had received only one steroid injection, the average time to ptosis onset was 2.7 months (range: 0-6, median: 2 months). No post-injection orbital fat prolapse was associated with ptosis. Eyelid crease height measurements were not available in the medical records. Seventeen patients underwent ptosis repair. Fourteen underwent one ptosis surgery, and three underwent more than one ptosis repair. Five patients

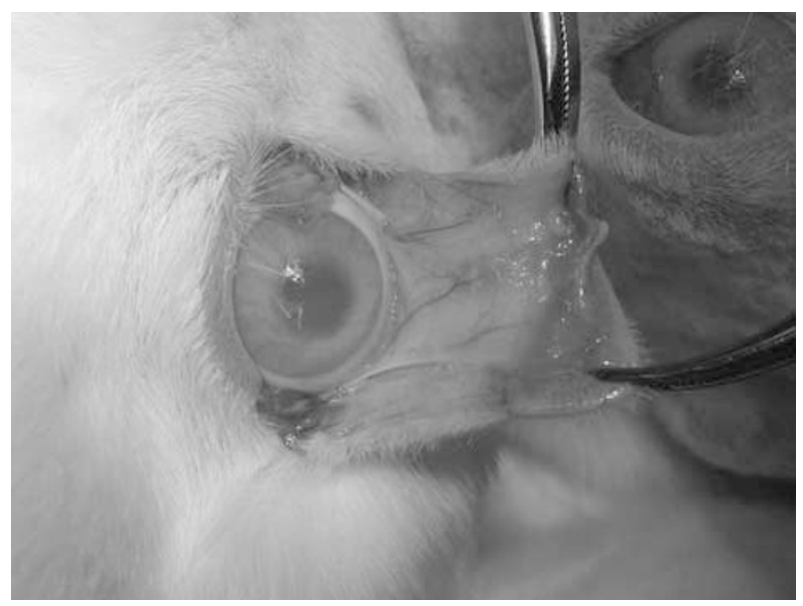

Figure 2 Dissection of levator tissues. The levator muscle and aponeurosis were harvested from the New Zealand rabbit. 
deferred ptosis repair. Of those patients undergoing ptosis repair, the mean MRD1 was $0.93 \mathrm{~mm}$ (range: 2-4 mm), and the mean postoperative MRD1 was $4.05 \mathrm{~mm}$ (range: $2-5 \mathrm{~mm}$ ). The mean preoperative levator function was $14.2 \mathrm{~mm}$ (range: $11-18 \mathrm{~mm}$ ), and the mean postoperative levator function was $15.3 \mathrm{~mm}$ (range: $15-16 \mathrm{~mm}$ ). In two eyes, the levator aponeurosis appeared thin intraoperatively. Disinsertion of the aponeurosis was never observed. Histopathologic examination of the tissues of two patients who underwent ptosis repair revealed no significant atrophy of the levator aponeurosis. There were no cases of spontaneous resolution or improvement of ptosis. The mean follow-up after ptosis repair was 14 months.

\section{Part 2}

Histopathologic analysis of the levator muscle and levator aponeurosis revealed no significant difference in atrophy or degree of inflammation in the tissues between experimental and control groups.

\section{Discussion}

Corticosteroids effectively treat a variety of systemic inflammatory medical conditions such as chronic pulmonary obstructive disease, asthma, and vasculitides. ${ }^{10,11}$ Ophthalmologists frequently used corticosteroids. Although quite effective, steroids carry many adverse ocular and systemic effects. Some ophthalmic preparations increase local delivery and minimize systemic side effects. ${ }^{12}$ Local injection of corticosteroids decreased inflammation more effectively than topical corticosteroids and tend to be safer than chronic systemic treatment. ${ }^{13}$

Steroids have been used in the treatment of posterior uveitis, ${ }^{6-8}$ dacryocystitis, and inflammatory and vascular lesions in the eyelid and orbit. ${ }^{2,6-8,13}$ Recently, ophthalmologists reported the use of orbital steroid injections for the treatment of Graves disease. ${ }^{14,15}$ Periocular injections of steroids can reduce diplopia rates, decrease extraocular muscle size ${ }^{15}$ and reverse Graves compressive optic neuropathy. ${ }^{14}$

Ocular hypertension, posterior subcapsular cataract formation, delayed wound healing, and infection are potential side effects of corticosteroids. ${ }^{10,16}$ Less common complications of corticosteroid include infectious crystalline keratopathy, corneal calcification, and tear film instability. ${ }^{16}$ In addition, decreased motility, ${ }^{3}$ subcutaneous fat atrophy, ${ }^{2}$ orbital fat atrophy, ${ }^{1}$ and orbital fat herniation ${ }^{4}$ after periocular injection of steroids may occur. Orbital rim fat atrophy has been reported following periocular corticosteroids for recurrent iritis and headaches. ${ }^{1}$ Linear subcutaneous fat atrophy has occurred after intralesional injection of corticosteroids to a periocular capillary hemangioma. ${ }^{2}$

It is unclear how posterior subtenon's triamcinolone injections affect periocular tissues. Studies ${ }^{12,13}$ have shown that long-acting steroids cause no significant inflammation or damage to extraocular muscles, to Tenon's capsule, or to the retrobulbar tissues histologically. Another study ${ }^{3}$ reported diplopia in a patient who had received two injections of subtenon's corticosteroid injection superiorly. Intraoperatively, there was no muscle restriction; however, the superior rectus muscle was thin and atrophic.

Posterior subtenon's steroid injections have gained increasing popularity, especially in the treatment of chronic inflammation and cystoid macular oedema. ${ }^{6-8}$ Rather than subject patients to systemic side effects of immunosuppression, posterior subtenon's or orbital floor injections of TAA allow the steroid to be placed in direct contact with the globe with effects lasting 3-4 weeks. ${ }^{7}$ Posterior subtenon's injections are not without risks. Rare, but serious complications include globe perforation, markedly elevated intraocular pressure that may require surgical removal of the corticosteroid or filtering surgery, scleral thinning, and infection. ${ }^{9}$

Recently, mild ptosis after topical corticosteroid administration has been reported. ${ }^{16}$ Although not proven, the topical steroid may have direct effects on Muller's muscle. In a study ${ }^{5}$ in which rhesus monkeys developed ptosis and mydriasis after administration of dexamethasone, the vehicle rather than the steroid itself (without the dexamethasone) was found to be myopathic.

Ptosis occurs in a small percentage of patients who had received subtenon's injection of corticosteroids for uveitis. ${ }^{6-8}$ In one study, ${ }^{8}$ only two cases of 58 eyes developed ptosis after posterior subtenon's steroid injection. In another study, ${ }^{7}$ mild persistent ptosis was noted in two eyes of 28 eyes. These patients had developed ptosis within the first month after injection. In both eyes, it appeared that there was disinsertion of the levator aponeurosis. In another study, ${ }^{6}$ only one patient out of 39 developed persistent ptosis following posterior subtenon's steroid injections for cystoid macular secondary to uveitis. This patient had undergone more than one steroid injection. An eyelid speculum had not been used in administration of the posterior subtenon's steroid injection in any of these studies.

In our study, a majority of patients received subtenon's steroid injections by two physicians (CB, JF), both of whom used similar methods. An eyelid speculum was used, and the steroid was directly placed into the subtenon's space. Although the onset of ptosis following steroid injection was variable, a majority of patients noted ptosis within the first few months of corticosteroid injection. In fact, three (22\%) patients noted ptosis between 
1 and 2 months after steroid injection. One (5\%) patient noted ptosis a few days after the steroid injection; this may have resulted from inadvertent steroid injection to the levator muscle. All of these patients had received only one steroid injection. The average time to ptosis was 13.9 months; however, this is most likely an overestimation of the actual time to ptosis development as patients may not have noted the development of ptosis until completion of uveitis treatment. Another factor accounting for delayed ptosis diagnosis is that many patients discussed the ptosis with the physician only when they came to regularly scheduled appointments. Most patients admitted to ptosis onset before their scheduled visits.

Although 22 patients were referred for ptosis evaluation after subtenon's steroid injection, it is possible that there were other patients who had less significant ptosis which were not noted. Unlike a recent study, ${ }^{17}$ we found no fat prolapse in our patients with ptosis after subtenon's steroid injection.

Steroid-induced myopathy has been described in human and animal skeletal muscles. ${ }^{11,18-20}$ Biopsies of skeletal muscle showed variation in muscle fibre size, atrophy, and necrosis. ${ }^{11,21}$ One study ${ }^{22}$ on steroidinduced myopathy in rabbits showed changes as early as $4 \mathrm{~h}$ after cortisone injections to one lower extremity. Muscle analyses were performed on the diaphragm, heart, and the noninjected lower extremity. Degenerative changes were noted on light microscopy after 2 days, and replacement of muscle fibres with vacuoles were noted 11-14 days after cortisone administration.

A few studies ${ }^{23,24}$ have observed the anatomic and histopathologic changes in ptosis, but none in patients with ptosis after corticosteroid exposure. One study ${ }^{23}$ analysed the changes in involutional ptosis. Myofibrils ranged from normal to atrophic. Electron microscopy revealed abnormalities in the mitochondria as well as dilated tubular systems. Kuwabara et $a l^{24}$ performed electron microscopy of eyelid muscles and noted reduction or absence of striated levator, vacuolation in muscle cells, and fibrosis of muscle cells in specimens with ptosis, regardless of aetiology, although there was a high degree of variability in the histopathologic changes.

Our prospective animal study sought to investigate whether histopathologic changes were present after steroid injection, excluding ptosis secondary to atrophy or damage to the levator tissues from inadvertent injection to the levator tissues. In our study, histopathologic examination did not reveal any significant inflammation or structural difference in the muscle or aponeurotic eyelid tissues compared to control tissues. One animal study ${ }^{18}$ on steroid-induced myopathy on rat soleus muscles showed muscle fibre necrosis and apoptotic myonuclei on electron microscopy after daily intraperitoneal TAA injections; another study $^{12}$ showed only minimal polymorphonuclear leukocyte infiltration in rabbit periocular tissues up to 2 months after subconjunctival and retrobulbar cortisone injection. In our study, no eyelid speculum was used at the time of subtenon's steroid injections in the animal study. We analysed tissues after only 18 days following steroid injection; this may have not been sufficient time to allow for significant changes in levator tissues to take place or for histopathologic changes to be detected. In addition, myofibril changes in the eyelid tissues are more difficult to analyse than other tissues. Masson trichrome stains and electron microscopy were deferred as significant differences were not noted on light microscopy.

Based on documented reports of ptosis and tissue changes after exposure to local corticosteroids, we conclude that posterior subtenon's triamcinolone injection may adversely affect nearby tissues. Based on previous clinical observations, the levator muscle or aponeurosis and/or the insertion site of the aponeurosis may be affected. Preoperative eyelid crease measurements may have been useful to assess this mechanism of ptosis; however, they were not available in all study patients. Although not investigated in this study, effects on Muller's muscle may also occur. Our study's limitations were: (1) the follow-up period of 18 days in the animal study which may have been an inadequate time to allow for ptosis to develop or any histopathologic changes to be evident in the eyelid tissues; (2) the small number of animals studied; (3) an eyelid speculum was used in most of the clinical cases but not in the animal studies. Biopsies of other adjacent tissues such as Muller's muscle and orbital tissues would have been useful. More long-term studies in addition to studies directly injecting steroids into the levator muscle are needed in order to better understand how ptosis occurs after exposure to steroids.

\section{Acknowledgements}

Supported in part by Research to Prevent Blindness, New York, New York and The University of Iowa Department of Ophthalmology and Visual Sciences Resident and Fellow Research Program. Work presented at the American Society of Ophthalmic Plastic and Reconstructive Surgery, Annual Fall Meeting, October 15, 2005.

\section{References}

1 Nozik RA. Orbital rim fat atrophy after repository periocular corticosteroid injection. Am J Ophthalmol 1976; 82(6): 928-930.

2 Droste PJ, Ellis FD, Sondhi N, Helveston EM. Linear subcutaneous fat atrophy after corticosteroid injection of 
periocular hemangiomas. Am J Ophthalmol 1988; 105(1): 65-69.

3 Raab EL. Limitation of motility after periocular corticosteroid injection. Am J Ophthalmol 1974; 78(6): 996-998.

4 Smith JR, George RK, Rosenbaum JT. Lower eyelid herniation of orbital fat may complicate periocular corticosteroid injection. Am J Ophthalmol 2002; 133(6): 845-847.

5 Newsome DA, Wong VG, Cameron TP, Anderson RR. Steroid-induced mydriasis and ptosis. Invest Ophthalmol 1971; 10(6): 424-429.

6 Yoshikawa K, Kotake S, Ichiishi A, Sasamoto Y, Kosaka S, Matsuda H. Posterior sub-tenon injections of repository corticosteroids in uveitis patients with cystoid macular edema. Jpn J Ophthalmol 1995; 39(1): 71-76.

7 Tanner V, Kanski JJ, Frith PA. Posterior sub-Tenon's triamcinolone injections in the treatment of uveitis. Eye 1998; 12: 679-685.

8 Lafranco Dafflon M, Tran VT, Guex-Crosier Y, Herbort CP. Posterior sub-Tenon's steroid injections for the treatments of posterior ocular inflammation: indications, efficacy and side effects. Graefe's Arch Clin Exp Ophthalmol 1999; 237(4): 289-295.

9 McGhee CNJ, Dean S, Danesh-Meyer H. Locally administered ocular corticosteroids. Drug Safety 2002; 25(1) 33-55.

10 Renfro L, Snow JS. Ocular effects of topical and systemic steroids. Oculocutaneous Dis 1992; 10(3): 505-512.

11 Decramer M. Respiratory muscles in COPD: regulation of trophical status. Verh K Acad Geneeskd Belg 2001; 63(6): $577-602$.

12 Leopold IH, Purnell JE, Cannon EJ, Steinmetz CG, McDonald PR. Local and systemic cortisone in ocular disease. Am J Ophthalmol 1951; 34: 361-371.
13 Rodger FC. Repository corticosteroid therapy in ophthalmic therapy in and practice. Br Ophthalmol 1965; 49: 298-306.

14 Ebner R. Dysthyroid optic neuropathy. Semin Ophthalmol 2002; 17(1): 18-21.

15 Ebner R, Devoto MH, Weil D, Bordaberry M, Mir C, Martinez $\mathrm{H}$ et al. Treatment of thyroid associated ophthalmopathy with periocular injections of triamcinolone. Br J Ophthalmol 2004; 88(1): 1380-1386.

16 McGhee CN, Ellerton CR. Complications of excimer laser photorefractive surgery. In: McGhee CN, Taylor HR, Gartry DS et al, (eds). Excimer Lasers in Ophthalmology, Principles and Practice. Martin Dunitz: London, 1997 pp 380-402 Chapter 23.

17 Dal Canto AJ, Downs-Kelly E, Perry JD. Ptosis and orbital fat prolapse after posterior sub-tenon's capsule triamcinolone injection. Ophthalmology 2005; 112: 1092-1097.

18 Lee MJ, Lee JS, Lee MC. Apoptosis of skeletal muscle on steroid-induced myopathy in rats. J Korean Med Sci 2001; 16: 467-474.

19 Kumar S. Steroid-induced myopathy following a single oral dose of prednisolone. Neurol India 2003; 51(4): 554-556.

20 Polsonetti BW, Joy SD, Laos LF. Steroid-induced myopathy in the ICU. Ann Pharmacother 2002; 36: 1741-1743.

21 Kanda F, Takatani K, Okuda S, Matsushita T, Chihara K. Preventive effects of insulinlike growth factor-I on steroid-induced muscle atrophy. Muscle Nerve 1999; 22(2): 213-217.

22 Afifi AK, Bergman RA. Steroid myopathy: a study of the evolution of the muscles lesion in rabbits. Johns Hopkins Med $J$ 1969; 124(2): 66-86.

23 Shore JW, McCord CD. Anatomic changes in involutional blepharoptosis. Am J Ophthalmol 1984; 98(1): 21-27.

24 Kuwabara T, Cogan DG, Johnson CC. Structure of the muscles of the upper eyelid. Arch Ophthalmol 1975; 93(11): 1189-1197. 